\title{
Reasoning about generalized intervals: Horn representability and tractability
}

\author{
Philippe Balbiani, Jean-François Condotta ${ }^{\dagger}$ Gérard Ligozat ${ }^{\ddagger}$
}

\begin{abstract}
This paper organizes the topologic forms of the possible relations between generalized intervals. Working out generalized interval algebra on the pattern of point algebra and interval algebra, it introduces the concept of Horn representability just as the one of convexity. It gives the class of Horn representable relations a simple characterization based on the concept of strong preconvexity. Adapting the propagation techniques designed to solve the networks of constraints between points or between intervals, it shows that the issue of consistency of a Horn representable generalized interval network can be solved in polynomial time by means of the weak path-consistency algorithm, a new incomplete algorithm for computing a minimal set of temporal constraints.
\end{abstract}

\section{INTRODUCTION}

It is hard to overemphasize the importance for computer science and artificial intelligence of the development of reasoning systems that are concerned with temporal information. The thing is that the nature of time raises grave difficulties for those who take on the matter of its representation. Without doubt, the model of the points designed by Vilain and Kautz [13] and the model of the intervals elaborated by Allen [1] are the better known models for reasoning about time. In these models, temporal information is represented by a network of constraints between a finite number of variables. An important matter is deciding consistency of a network. Concerning points, Ladkin and Maddux [6] prove that the issue of consistency of a point network can be solved in polynomial time by means of the path-consistency algorithm. Relating to intervals, Vilain and Kautz [13] demonstrate that deciding consistency of an interval network becomes NP-complete.

*Laboratoire d'informatique de Paris-Nord, 99 avenue Jean-Baptiste Clément, F-93430 Villetaneuse

${ }^{\dagger}$ Institut de recherche en informatique de Toulouse, 118 route de Narbonne, F-31062 Toulouse Cedex 4

$\ddagger$ Laboratoire d'informatique pour la mécanique et les sciences de l'ingénieur, BP 133, F-91403 Orsay 
Therefore, the question of characterizing tractable subclasses of interval algebra has been considered. Nebel and Bürckert [10] give a definitive answer to the question of which subclasses among those which contain base relations are tractable. To be more precise, the subclass of Horn representable relations is the unique maximal tractable subclass having this property. Moreover, deciding consistency can be accomplished by using the path-consistency algorithm. Horn representability is a syntactic concept, in view of the fact that Horn representable relations can be described by Horn clauses in a suitable language. Ligozat [8] produces a simple characterization of the same class in terms of preconvex relations. Preconvexity is a geometric concept, for the simple reason that preconvex relations can be roughly described as convex relations with some lower dimensional base relations taken out.

Working out generalized interval algebra on the pattern of point algebra and interval algebra, Ligozat [7] organizes the topologic forms of the possible relations between generalized intervals. A problem is that the coincidence between the syntactic concept of Horn representability and the geometric concept of preconvexity does not hold any longer, as Ligozat [8] notices in the context of generalized interval algebra, because the subclass of Horn representable relations is a proper subset of the set of all preconvex relations. A further complication is that, as Balbiani, Condotta and Fariñas del Cerro [3] remark in the context of rectangle algebra, the set of all preconvex relations is not a subclass in the usual sense, given that it is not closed for intersection. This leads them to define a restricted geometric notion, the concept of strong preconvexity, which has this closure property.

In the context of generalized interval algebra as well, it is interesting to consider the tractability issues both from the syntactic point of view and the geometric one. A primary goal of this paper is to give the class of Horn representable relations between generalized intervals a simple characterization in terms of strongly preconvex relations. An outcome of this characterization is that it allows to demonstrate that the issue of consistency of a Horn representable generalized interval network can be solved in polynomial time by means of the weak path-consistency algorithm, a new incomplete algorithm for computing a minimal set of temporal constraints. Successive sections are arranged along the following lines. Section 2 introduces the relational algebra of generalized intervals. We devote the whole section 3 to the syntactic concept of Horn representability. In sections 4,5 and 6 , we identify the geometric concepts of convexity, weak preconvexity and strong preconvexity. Section 7 focuses on the issue of consistency of a generalized interval network.

\section{GENERALIZED ALGEBRA}

Given a model of time consisting of the totally ordered set of all rational numbers, a generalized interval is a list of $p$ rational numbers, with the first number less than the second, the second less than the third, etc. We will use $x, y$, z, etc, for these, assuming for any list $x$ of $p$ rational numbers, the first number is denoted by $x_{1}$, the second by $x_{2}$, etc. Such lists of $p$ rational numbers are also sometimes called $p$-intervals. We want 
to formalize the notion of binary relation between a $p$-interval and a $q$-interval for any $p, q \geq 1$. To keep things concrete, we will confine ourselves to results about the notion of binary relation between two $p$-intervals for some $p \geq 1$. Extending these to the remaining cases is a simple matter. In order to formalize the position of $x$ with respect to $y$ we have to decompose the set of all rational numbers into subsets. If we define $y_{0}$ as $-\infty$ and $y_{p+1}$ as $+\infty$ then the numbers $y_{1}, \ldots, y_{p}$ clearly define a partition of this set into $2 \times p+1$ zones numbered from 0 to $2 \times p$ such that:

- For all $i \in\{0, \ldots, p\}$, zone $2 \times i$ is $] y_{i}, y_{i+1}[$;

- For all $i \in\{1, \ldots, p\}$, zone $2 \times i-1$ is $\left\{y_{i}\right\}$.



Figure 1: A 3-interval $x=\left(x_{1}, x_{2}, x_{3}\right)$.

Obviously, each rational number belongs to exactly one zone. This shows that the position of $x$ with respect to $y$ is a sequence of $p$ zones which specifies for each $i \in$ $\{1, \ldots, p\}$ which zone the number $x_{i}$ belongs to. Let $\mathcal{Q}$ be the set of all sequences of $p$ zones. We will use $a, b, c$, etc, for these, assuming for any sequence $a$ of $p$ zones, the first zone is denoted by $a_{1}$, the second by $a_{2}$, etc. If we define $a_{0}$ as 0 and $a_{p+1}$ as $2 \times p$ then:

- $a$ is a position between generalized intervals iff, for all $i \in\{1, \ldots, p\}, a_{i} \in$ $\left[\left[a_{i-1}, a_{i+1}\right]\right]$, assuming for any pair $a, b$ of integers, $[[a, b]]$ is the largest integer interval with even endpoints and contained in $[a, b]$.

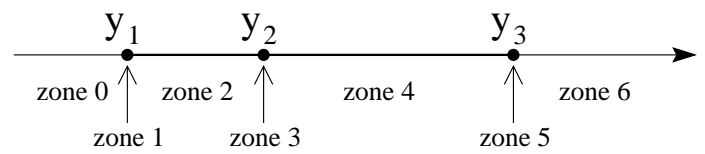

Figure 2: The 7 zones defined by a 3 -interval $y=\left(y_{1}, y_{2}, y_{3}\right)$.

Let $\mathcal{P}$ be the set of all positions. Positions are also called basic relations. They constitute the exhaustive list of the possible relations between generalized intervals. For example, if $\left.x_{1} \in\right] y_{0}, y_{1}\left[, x_{2} \in\right] y_{0}, y_{1}\left[\right.$ and $\left.x_{3} \in\right] y_{2}, y_{3}[$ then the position of $x$ with respect to $y$ is the sequence $(0,0,4)$ and if $\left.x_{1} \in\right] y_{0}, y_{1}\left[, x_{2}=y_{1}\right.$ and $\left.x_{3} \in\right] y_{2}, y_{3}[$ then the position of $x$ with respect to $y$ is the sequence $(0,1,4)$. In order to represent indefinite information, we allow the binary relation between two generalized intervals to be any subset of the set of all basic relations. We will use $\alpha, \beta$, $\gamma$, etc, for these. In the relational approach to temporal reasoning, the operations of inverse and composition play an important role. The inverse of $a$, denoted by $a^{-1}$, is the position $b$ such that, for all $i \in\{1, \ldots, p\}$ : 
- For all $j \in\{0, \ldots, p\}$, if $2 \times i-1 \in\left[\left[a_{j}, a_{j+1}\right]\right]$ then $b_{i}=2 \times j$;

- For all $j \in\{1, \ldots, p\}$, if $2 \times i-1=a_{j}$ then $b_{i}=2 \times j-1$.

The composition of $a$ and $b$, denoted by $a ; b$, is the set of all positions $c$ such that, for all $i \in\{1, \ldots, p\}$ :

- For all $j \in\{0, \ldots, p\}$, if $a_{i}=2 \times j$ then $c_{i} \in\left[\left[b_{j}, b_{j+1}\right]\right]$;

- For all $j \in\{1, \ldots, p\}$, if $a_{i}=2 \times j-1$ then $c_{i}=b_{j}$.

For instance, the inverses of $(0,0,4)$ and $(0,1,4)$ are the positions $(4,4,6)$ and $(3,4,6)$ whereas the composition of $(0,0,4)$ and $(0,1,4)$ is the set $\{(0,0,2),(0,0,3),(0,0,4)\}$ of positions. Since binary relations between generalized intervals are sets of basic relations, the operations of inverse and composition are extended as follows. The inverse of $\alpha$, denoted by $\alpha^{-1}$, is $\left\{a^{-1}: a \in \alpha\right\}$. The composition of $\alpha$ and $\beta$, denoted by $\alpha ; \beta$, is $\bigcup\{a ; b: a \in \alpha \& b \in \beta\}$. This brings us to the question of whether these definitions capture the intended meaning of the operations involved. Let $x \alpha y$ mean that the position of $x$ with respect to $y$ belongs to $\alpha$. Ligozat [7] shows that the operations of inverse and composition have the following important properties:

- $x \alpha^{-1} y$ iff $y \alpha x$

- $x \alpha ; \beta y$ iff there is a generalized interval $z$ such that $x \alpha z$ and $z \beta y$.

This proves a simple but fundamental result:

- The algebra $\left(2^{\mathcal{P}}, \cup, \cap,-, \emptyset, \mathcal{P},{ }^{-1}, ;,\{(1, \ldots, 2 \times p-1)\}\right)$ is a relational algebra.

\section{HORN REPRESENTABILITY}

Horn representable relations correspond to particular sets of clauses. Clauses are built up from $p$ variables $u_{1}, \ldots, u_{p}$ and $p$ variables $v_{1}, \ldots, v_{p}$ using the arithmetical symbols $<,=,>, \leq, \neq$ and $\geq$. A literal is any expression of the form $u_{i} \Re v_{j}$, where $i, j \in$ $\{1, \ldots, p\}$ and $\Re$ is an arithmetical symbol. A set of literals is a Horn clause iff it contains zero or exactly one positive literal, assuming for any $i, j \in\{1, \ldots, p\}$, the literals $u_{i}<v_{j}, u_{i}=v_{j}, u_{i}>v_{j}, u_{i} \leq v_{j}$ and $u_{i} \geq v_{j}$ are positive and the literal $u_{i} \neq v_{j}$ is negative. For example, the clauses $\left\{u_{2}=v_{1}, u_{3} \neq v_{2}\right\}$ and $\left\{u_{2}=\right.$ $\left.v_{1}, u_{3} \neq v_{3}\right\}$ are Horn clauses whereas the clauses $\left\{u_{2}<v_{1}, u_{3} \neq v_{2}, u_{3}=v_{3}\right\}$ and $\left\{u_{2}>v_{1}, u_{3}=v_{2}, u_{3} \neq v_{3}\right\}$ are not Horn clauses. A definite clause contains exactly one positive literal and zero or more negative literals. A positive unit clause is a definite clause containing no negative literal. Nebel and Bürckert [10] consider only Horn clauses using the arithmetical symbols $=, \leq, \neq$ and $\geq$. It is straightforward to prove that every Horn clause using the arithmetical symbols $<,=,>, \leq, \neq$ and $\geq$ is equivalent to a couple of Horn clauses using the arithmetical symbols $=, \leq, \neq$ and $\geq$. To define Horn representable relations we need to evaluate variables occurring in the 
given literals of a clause. We shall say that $a$ validates the literal $u_{i} \Re v_{j}$ iff $a_{i} \Re(2 \times$ $j-1)$. For instance, $(0,1,3)$ and $(0,1,5)$ validate the literal $u_{2}=v_{1}$, seeing that $1=(2 \times 1-1)$. In this spirit, a basic relation validates a clause iff it validates at least one literal of the clause. For example, $(0,1,3)$ and $(0,1,5)$ validate the clauses $\left\{u_{2}=\right.$ $\left.v_{1}, u_{3} \neq v_{2}\right\}$ and $\left\{u_{2}=v_{1}, u_{3} \neq v_{3}\right\}$. Then we can define the Horn representable relations as follows:

- $\alpha$ is Horn representable iff there is a set $\mathcal{S}$ of Horn clauses such that exactly the basic relations of $\alpha$ validate every clause of $\mathcal{S}$.

One says that $\mathcal{S}$ is a Horn representation of $\alpha$. To illustrate the truth of this, the binary relations $\{(0,0,4),(0,1,3),(0,1,4),(0,1,5)\}$ and $\{(0,1,3),(0,1,4),(0,1,5),(0,2$, 4) $\}$ are Horn representable or to be more precise, the Horn clauses $\left\{u_{1}<v_{1}\right\},\left\{u_{2} \leq\right.$ $\left.v_{1}\right\},\left\{u_{3} \geq v_{2}\right\},\left\{u_{3} \leq v_{3}\right\},\left\{u_{2}=v_{1}, u_{3} \neq v_{2}\right\}$ and $\left\{u_{2}=v_{1}, u_{3} \neq v_{3}\right\}$ constitute a Horn representation of the former binary relation whereas the Horn clauses $\left\{u_{1}<v_{1}\right\}$, $\left\{u_{2} \geq v_{1}\right\},\left\{u_{2}<v_{2}\right\},\left\{u_{3} \geq v_{2}\right\},\left\{u_{3} \leq v_{3}\right\},\left\{u_{2}=v_{1}, u_{3} \neq v_{2}\right\},\left\{u_{2}=v_{1}, u_{3} \neq\right.$ $\left.v_{3}\right\}$ constitute a Horn representation of the latter binary relation. It is not necessarily the case that the line of reasoning suggested by Nebel and Bürckert [10] within the context of Horn representable relations between intervals applies to Horn representable relations between generalized intervals when $p \geq 3$. In particular, although the reader may easily verify that the set of all Horn representable relations between generalized intervals is closed for intersection and inverse, there is no evidence that the set of all Horn representable relations between generalized intervals is closed for composition when $p \geq 3$. Therefore, we are not in a position to give any sort of proof that the set of all Horn representable relations between generalized intervals constitutes a subclass of the generalized interval algebra when $p \geq 3$.

\section{CONVEXITY}

To define the set of all convex relations, it is helpful to first arrange in ascending order the sequences of $p$ zones. Let $a \preceq b$ mean that $a_{i} \leq b_{i}$ for all $i \in\{1, \ldots, p\}$. For instance, $(0,0,3) \preceq(0,1,5)$ and $(0,1,3) \preceq(0,2,5)$. As a product of chains, it is easily shown that $(\mathcal{Q}, \preceq)$ is a distributive lattice. $(\mathcal{P}, \preceq)$ is also a distributive lattice, because $(\mathcal{P}, \preceq)$ is a sublattice of $(\mathcal{Q}, \preceq)$. The interval bounded by $a$ and $b$, denoted by $[a, b]$, is the binary relation $\{c: a \preceq c$ and $c \preceq b\}$. This leads us to make the following definition:

- $\alpha$ is convex iff there are positions $a, b$ such that $\alpha=[a, b]$.

To illustrate the truth of this, the binary relation $\{(0,1,3),(0,1,4),(0,1,5)\}$ is convex. Clearly, convex relations are Horn representable or to be more precise, if $\alpha$ is convex then there is a set $\mathcal{S}$ of positive unit clauses such that $\mathcal{S}$ is a Horn representation of $\alpha$. For instance, the positive unit clauses $\left\{u_{1}<v_{1}\right\},\left\{u_{2} \leq v_{1}\right\},\left\{u_{3} \geq v_{2}\right\}$, $\left\{u_{3} \leq v_{3}\right\}$ constitute a Horn representation of the interval bounded by $(0,0,3)$ and $(0,1, \overline{5})$ whereas the positive unit clauses $\left\{u_{1}<v_{1}\right\},\left\{u_{2} \geq v_{1}\right\},\left\{u_{2}<v_{2}\right\}$, 


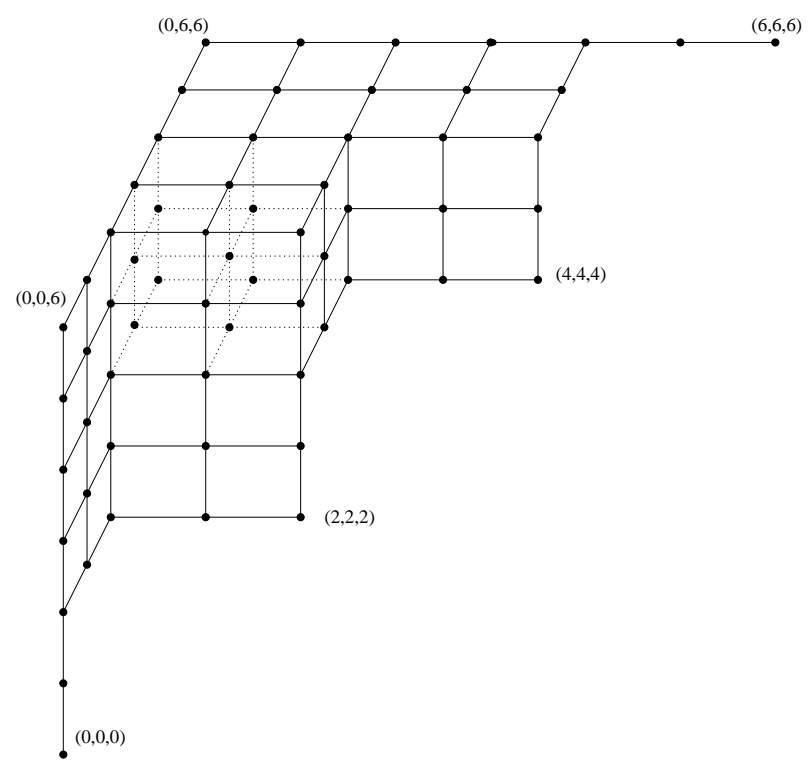

Figure 3: The lattice $(\mathcal{P}, \preceq)$ for $p=3$.

$\left\{u_{3} \geq v_{2}\right\},\left\{u_{3} \leq v_{3}\right\}$ constitute a Horn representation of the interval bounded by $(0,1,3)$ and $(0,2,5)$. In general, the converse is false, Horn representable relations need not be convex. For a counterexample, take the case of the Horn representable relations $\{(0,0,4),(0,1,3),(0,1,4),(0,1,5)\}$ and $\{(0,1,3),(0,1,4),(0,1,5),(0,2,4)\}$. Evidently, the set of all convex relations is closed for intersection. In particular, the set of all convex relations containing $\alpha$ contains a least element, denoted by $I(\alpha)$, the convex closure of $\alpha$. Ligozat [7] demonstrates that the convex closure has the following important properties:

$-I\left(\alpha^{-1}\right)=I(\alpha)^{-1}$

- $I(\alpha ; \beta)=I(\alpha) ; I(\beta)$.

The conclusion can be summarized as follows: the set of all convex relations is closed for inverse and composition. Therefore, the set of all convex relations constitutes a subclass of the generalized interval algebra: the convex class.

\section{WEAK PRECONVEXITY}

To define the set of all weakly preconvex relations, we have to bring in the operations of topologic closure and dimension as follows. The topologic closure of $a$, denoted by $C(a)$, is the set of all positions $b$ such that, for all $i \in\{1, \ldots, p\}$, either $b_{i}=a_{i}$ 
or $\left|b_{i}-a_{i}\right|=1$ and $b_{i}$ is odd. For instance, the topologic closure of $(0,1,4)$ is the binary relation $\{(0,1,3),(0,1,4),(0,1,5)\}$. The dimension of $a$, denoted by $\operatorname{dim}(a)$, is $p-\Sigma\left\{a_{i} \bmod 2: i \in\{1, \ldots, p\}\right\}$. For example, the dimension of $(0,1,4)$ is 2 . Seeing that binary relations between generalized intervals are sets of basic relations, we extend the operations of topologic closure and dimension as follows. The topologic closure of $\alpha$, denoted by $C(\alpha)$, is $\bigcup\{C(a): a \in \alpha\}$. The dimension of $\alpha$, denoted by $\operatorname{dim}(\alpha)$, is $\sup \{\operatorname{dim}(a): a \in \alpha\}$. Ligozat [9] proves that the following conditions are equivalent:

- $C(\alpha)$ is convex;

$-I(\alpha) \subseteq C(\alpha)$

$-\operatorname{dim}(I(\alpha) \backslash \alpha)<\operatorname{dim}(\alpha)$.

This justifies the role played by topologic closure in the following definition:

- $\alpha$ is weakly preconvex iff $C(\alpha)$ is convex iff $I(\alpha) \subseteq C(\alpha)$ iff $\operatorname{dim}(I(\alpha) \backslash \alpha)<$ $\operatorname{dim}(\alpha)$.

For instance, the binary relations $\{(0,0,4),(0,1,3),(0,1,5)\}$ and $\{(0,1,3),(0,1,5)$, $(0,2,4)\}$ are weakly preconvex or more exactly, the topologic closure of the former binary relation is the interval bounded by $(0,0,4)$ and $(0,1,5)$ whereas the topologic closure of the latter binary relation is the interval bounded by $(0,1,3)$ and $(1,3,5)$. It is clear that convex relations are weakly preconvex. This brings us to the question of whether Horn representable relations are weakly preconvex. Suppose $\alpha$ is Horn representable, we show it is weakly preconvex. By our definition of Horn representability, we know that there is a set $\mathcal{S}$ of Horn clauses such that $\mathcal{S}$ is a Horn representation of $\alpha$. Let $\mathcal{S}^{\circ}$ consist of all positive unit clauses of $\mathcal{S}$. With no loss of generality, suppose, for all $i, j \in\{1, \ldots, p\}$, if $u_{i} \neq v_{j}$ appears in some clause of $\mathcal{S} \backslash \mathcal{S}^{\circ}$ then $u_{i}=v_{j}$ is not a consequence of $\mathcal{S}^{\circ}$. Let $\gamma$ be the binary relation which elements are exactly the basic relations validating every clause of $\mathcal{S}^{\circ}$. Since $\mathcal{S}^{\circ} \subseteq \mathcal{S}$, then $\alpha \subseteq \gamma$. Since $\mathcal{S}^{\circ}$ is a set of positive unit clauses, then $\gamma$ is convex. It follows that $I(\alpha) \subseteq \gamma$. Let us show that $\gamma \subseteq C(\alpha)$. Consider the basic relation $a$ of $\gamma$. It follows that $a$ validates every clause of $\mathcal{S}^{\circ}$. Then we can define a basic relation $b$ of $\alpha$ such that $a \in C(b)$ as follows. Let $i \in\{1, \ldots, p\}$. If $a_{i}$ is even then there is $j \in\{0, \ldots, p\}$ such that $a_{i}=2 \times j$ and let $b_{i}=2 \times j$. Otherwise $a_{i}$ is odd and there is $j \in\{1, \ldots, p\}$ such that $a_{i}=2 \times j-1$. If $u_{i}=v_{j}$ is a consequence of $\mathcal{S}^{\circ}$ then let $b_{i}=2 \times j-1$. Otherwise $u_{i} \leq v_{j}$ is not a consequence of $\mathcal{S}^{\circ}$ or $u_{i} \geq v_{j}$ is not a consequence of $\mathcal{S}^{\circ}$. In the former case let $b_{i}$ $=2 \times j$ whereas in the latter case let $b_{i}=2 \times(j-1)$. The reader may easily verify that $b$ is a basic relation of $\alpha$ such that $a \in C(b)$. This gives us the following result: $\gamma$ $\subseteq C(\alpha)$. It follows that $I(\alpha) \subseteq C(\alpha)$, hence $\alpha$ is weakly preconvex. From all this it follows that:

Theorem 1 If $\alpha$ is Horn representable then $\alpha$ is weakly preconvex. 
Although Ligozat [8] shows that weakly preconvex relations between intervals are Horn representable, weakly preconvex relations between generalized intervals need not be Horn representable when $p \geq 3$, unfortunately. Take, for example, the case of the weakly preconvex relations $\{(0,0,4),(0,1,3),(0,1,5)\}$ and $\{(0,1,3),(0,1,5),(0,2$, 4)\}. Ligozat [9] demonstrates that the topologic closure has the following important properties:

$$
\begin{aligned}
& \text { - } C\left(\alpha^{-1}\right) \supseteq C(\alpha)^{-1} ; \\
& \text { - } C(\alpha ; \beta) \supseteq C(\alpha) ; C(\beta) .
\end{aligned}
$$

The interesting result is: the set of all weakly preconvex relations is closed for inverse and composition. Although Ligozat [8] proves that the set of all weakly preconvex relations between intervals is closed for intersection, the reader may easily verify that the set of all weakly preconvex relations between generalized intervals is not closed for intersection when $p \geq 3$. Consider, for instance, the weakly preconvex relations $\{(0,0,4),(0,1,3),(0,1,5)\}$ and $\{(0,1,3),(0,1,5),(0,2,4)\}$. Therefore, the set of all weakly preconvex relations between generalized intervals does not constitutes a subclass of the generalized interval algebra when $p \geq 3$.

\section{STRONG PRECONVEXITY}

Assume $p \geq 3$. The trouble with the set of all weakly preconvex relations is that it not closed for intersection with convex relations. One has only to consider the weakly preconvex relations $\{(0,0,4),(0,1,3),(0,1,5)\}$ and $\{(0,1,3),(0,1,5),(0,2,4)\}$ and the convex relation $\{(0,1,3),(0,1,4),(0,1,5)\}$. This justifies the role played by intersection with convex relations in the following definition:

- $\alpha$ is strongly preconvex iff, for all binary relation $\beta$, if $\beta$ is convex then $\alpha \cap \beta$ is weakly preconvex.

To illustrate the truth of this, the binary relations $\{(0,0,4),(0,1,3),(0,1,4),(0,1,5)\}$ and $\{(0,1,3),(0,1,4),(0,1,5),(0,2,4)\}$ are strongly preconvex. Clearly, strongly preconvex relations are weakly preconvex. Let us see if there is any connection between Horn representability and strong preconvexity. Suppose $\alpha$ is Horn representable, we demonstrate it is strongly preconvex. By our definition of Horn representability, we know that there is a set $\mathcal{S}(\alpha)$ of Horn clauses such that $\mathcal{S}(\alpha)$ is a Horn representation of $\alpha$. If $\alpha$ is not strongly preconvex then there is a binary relation $\beta$ such that $\beta$ is convex and $\alpha \cap \beta$ is not weakly preconvex. Since $\beta$ is convex, then it is Horn representable. In particular, there is a set $\mathcal{S}(\beta)$ of positive unit clauses such that $\mathcal{S}(\beta)$ is a Horn representation of $\beta$. Furthermore, the reader may easily verify that $\mathcal{S}(\alpha) \cup \mathcal{S}(\beta)$ is a Horn representation of $\alpha \cap \beta$. By theorem $1, \alpha \cap \beta$ is weakly preconvex, and this is impossible. These considerations prove:

Theorem 2 If $\alpha$ is Horn representable then $\alpha$ is strongly preconvex. 
Now suppose $\alpha$ is strongly preconvex, we prove it is Horn representable. Since $I(\alpha)$ is convex, then it is Horn representable. In particular, there is a set $\mathcal{S}(I(\alpha))$ of positive unit clauses such that $\mathcal{S}(I(\alpha))$ is a Horn representation of $I(\alpha)$. Consider the basic relation $a$ of $I(\alpha)$. Suppose $a \notin \alpha$. Let $\alpha_{a}=\left\{b\right.$ : for all $i \in\{1, \ldots, p\}$, if $a_{i}$ is odd then $b_{i}=a_{i}$. Obviously, $a \in \alpha_{a}$. Furthermore, the reader may easily verify that $\alpha_{a}$ is convex. It follows that $\alpha_{a}$ is Horn representable. What is more, there is a set $\mathcal{S}\left(\alpha_{a}\right)$ $=\left\{\left\{u_{i_{1}}=v_{j_{1}}\right\}, \ldots,\left\{u_{i_{M}}=v_{j_{M}}\right\}\right\}$ of positive unit clauses using the arithmetical symbol $=$ such that $\mathcal{S}\left(\alpha_{a}\right)$ is a Horn representation of $\alpha_{a}$. Let $\beta_{a}=\alpha \cap \alpha_{a}$. Since $\alpha$ is strongly preconvex, then $\beta_{a}$ is weakly preconvex. It follows that $I\left(\beta_{a}\right) \subseteq C\left(\beta_{a}\right)$. Let us demonstrate that $a \notin I\left(\beta_{a}\right)$. Suppose $a \in I\left(\beta_{a}\right)$, we derive a contradiction. Since $I\left(\beta_{a}\right) \subseteq C\left(\beta_{a}\right)$, then $a \in C\left(\beta_{a}\right)$ and there is a basic relation $b$ of $\beta_{a}$ such that $a \in C(b)$. Since $b \in \beta_{a}$, then $b \in \alpha$ and $b \in \alpha_{a}$. It follows that $b \neq a$ and, for all $i \in\{1, \ldots, p\}$, if $a_{i}$ is odd then $b_{i}=a_{i}$. Since $a \in C(b)$, then, for all $i \in\{1, \ldots, p\}$, either $a_{i}=b_{i}$ or $\left|a_{i}-b_{i}\right|=1$ and $a_{i}$ is odd, hence if $a_{i}$ is even then $b_{i}=a_{i}$. It follows that $b=a$, a contradiction. This gives us the following result: $a \notin I\left(\beta_{a}\right)$. Since $I\left(\beta_{a}\right)$ is convex, then $I\left(\beta_{a}\right)$ is Horn representable. To be more precise, there is a nonempty set $\mathcal{S}\left(I\left(\beta_{a}\right)\right)$ $=\left\{\left\{u_{k_{1}} \Re_{l_{1}} v_{m_{1}}\right\}, \ldots,\left\{u_{k_{N}} \Re_{l_{N}} v_{m_{N}}\right\}\right\}$ of positive unit clauses such that $\mathcal{S}\left(I\left(\beta_{a}\right)\right)$ is a Horn representation of $I\left(\beta_{a}\right)$. Let $\gamma_{a}=\alpha_{a} \backslash I\left(\beta_{a}\right)$ and $\delta_{a}=I(\alpha) \backslash \gamma_{a}$. It follows that $\delta_{a}$ is Horn representable. Ultimately, then, the set $\mathcal{S}\left(\delta_{a}\right)=\mathcal{S}(I(\alpha)) \cup\left\{\left\{u_{i_{1}} \neq\right.\right.$ $\left.\left.v_{j_{1}}, \ldots, u_{i_{M}} \neq v_{j_{M}}, u_{1} \Re_{l_{1}} v_{m_{1}}\right\}, \ldots,\left\{u_{i_{1}} \neq v_{j_{1}}, \ldots, u_{i_{M}} \neq v_{j_{M}}, u_{k_{N}} \Re_{l_{N}} v_{m_{N}}\right\}\right\}$ of definite clauses is a Horn representation of $\delta_{a}$. All in all, let $\mathcal{S}=\bigcup\left\{\mathcal{S}\left(\delta_{a}\right): a \in\right.$ $I(\alpha) \backslash \alpha\}$. The reader may easily verify that exactly the basic relations of $\alpha$ validate every clause of $\mathcal{S}$. It follows that $\alpha$ is Horn representable. Hence we have:

Theorem 3 If $\alpha$ is strongly preconvex then $\alpha$ is Horn representable.

\section{GENERALIZED NETWORKS}

Assume $p \geq 3$. A generalized interval network is a structure of the form $(n, M)$ where $n \geq 1$ and $M$ is a square $n \times n$ matrix with entries in $2^{\mathcal{P}}$. Hence $M$ isa function assigning, for all $i, j \in\{1, \ldots, n\}$, a subset $M(i, j)$ of $\mathcal{P}$, i.e. a binary relation. A tuple $(x(1), \ldots, x(n))$ of generalized intervals is called a (maximal) solution of $(n, M)$ iff, for all $i, j \in\{1, \ldots, n\}$, there is a basic relation $a$ of (maximal dimension in) $M(i, j)$ such that $x(i)$ a $x(j) .(n, M)$ is (maximally) consistent iff it possesses a (maximal) solution. In what follows we assume that all our generalized interval networks satisfy the following conditions:

$$
\begin{aligned}
& \text { - For all } i \in\{1, \ldots, n\}, M(i, i)=\{(1, \ldots, 2 \times p-1)\} \text {; } \\
& \text { - For all } i, j \in\{1, \ldots, n\}, M(i, j)=M(j, i)^{-1} .
\end{aligned}
$$

It is a well-known fact that by applying the following algorithm — the path-consistency algorithm - we obtain in polynomial time an equivalent generalized interval network: 
- Successively replace, for all pairwise distinct $i, j, k \in\{1, \ldots, n\}$, the constraints $M(i, k)$ and $M(k, i)$ by the constraints $M(i, k) \cap(M(i, j) ; M(j, k))$ and $M(k$, i) $\cap(M(k, j) ; M(j, i))$.

We make use of this in the following definition:

- $(n, M)$ is path-consistent iff, for all pairwise distinct $i, j, k \in\{1, \ldots, n\}, M(i$, $k) \subseteq M(i, j) ; M(j, k)$ and $M(k, i) \subseteq M(k, j) ; M(j, i)$.

An important matter is deciding consistency of a generalized interval network. It would be naive to suppose that constraints between generalized intervals can be expressed in terms of constraints between intervals, for the simple reason that although Ligozat [9] shows that if a weakly preconvex generalized interval network is path-consistent then either it contains the empty constraint or it is maximally consistent, the issue of consistency of a weakly preconvex generalized interval network is NP-complete. The fact of the matter is that the issue of consistency of an interval network is polynomial-time reducible to the issue of consistency of a weakly preconvex generalized interval network. Consider the interval network $(n, M)$. Then we can define the weakly preconvex generalized interval network $\left(n^{\prime}, M^{\prime}\right)$ as follows. Let $n^{\prime}=n \times(n+1)$. For all $i, j \in$ $\{1, \ldots, n\}$, let:

$$
\begin{aligned}
- & M^{\prime}(i, j)=\left\{\left(a_{1}, a_{2}, 5, \ldots, 2 \times p-1\right):\left(a_{1}, a_{2}\right) \in I(M(i, j))\right\} ; \\
- & M^{\prime}(i, n+i+(j-1) \times n)=\left\{\left(a_{1}, a_{2}, 5, \ldots, 2 \times p-1\right):\left(a_{1}, a_{2}\right) \in M(i, j)\right\} \cup \\
& \left\{\left(b_{1}, b_{2}, 6, \ldots, 2 \times p\right): b_{1}, b_{2} \in\{0,2,4\} \text { and } b_{1} \leq b_{2}\right\} ; \\
- & M^{\prime}(n+i+(j-1) \times n, j)=\{(1,3,5, \ldots, 2 \times p-1)\} .
\end{aligned}
$$

The reader may easily verify that $\left(n^{\prime}, M^{\prime}\right)$ is consistent iff $(n, M)$ is consistent. All this goes to show that:

Theorem 4 The issue of consistency of a weakly preconvex generalized interval network is NP-complete.

This polynomial-time reducibility of the issue of consistency of an interval network to the issue of consistency of a weakly preconvex generalized interval network serves to illustrate the role played by intersection with convex relations in the following definition:

- $(n, M)$ is weakly path-consistent iff, for all pairwise distinct $i, j, k \in\{1, \ldots, n\}$, $M(i, k) \subseteq I(M(i, j) ; M(j, k))$ and $M(k, i) \subseteq I(M(k, j) ; M(j, i))$.

From all the evidence it is clear that by applying the following algorithm - the weak path-consistency algorithm - we obtain in polynomial time an equivalent weakly pathconsistent network:

- Successively replace, for all pairwise distinct $i, j, k \in\{1, \ldots, n\}$, the constraints $M(i, k)$ and $M(k, i)$ by the constraints $M(i, k) \cap I(M(i, j) ; M(j, k))$ and $M(k$, i) $\cap I(M(k, j) ; M(j, i))$. 
We first observe that path-consistent generalized interval networks are weakly pathconsistent. In general, the converse is false, weakly path-consistent generalized interval networks need not be path-consistent. Let us demonstrate that the problem of deciding consistency of a generalized interval network can be solved in polynomial time by means of the weak path-consistency algorithm if only Horn representable relations are used. Consider the Horn representable generalized interval network $(n, M)$. Seeing that Horn representable relations are strongly preconvex, $(n, M)$ is a strongly preconvex generalized interval network. In view of the fact that the set of all strongly preconvex relations is closed for intersection with convex relations, it is beyond question that by applying the weak path-consistency algorithm, we obtain in polynomial time an equivalent weakly path-consistent strongly preconvex generalized interval network $\left(n, M^{\prime}\right)$. Then we can define the convex generalized interval network $\left(n, M^{\prime \prime}\right)$ as follows. For all $i, j \in\{1, \ldots, n\}$, let $M^{\prime \prime}(i, j)=I\left(M^{\prime}(i, j)\right)$. Given that the set of all convex relations is closed for composition, $\left(n, M^{\prime \prime}\right)$ is a path-consistent convex generalized interval network. Seeing that convex relations are weakly preconvex, $\left(n, M^{\prime \prime}\right)$ is a path-consistent weakly preconvex generalized interval network. In this respect, either it contains the empty constraint or it is maximally consistent. The former case implies that $\left(n, M^{\prime}\right)$ contains the empty constraint, hence $(n, M)$ is not consistent. The latter case implies that $\left(n, M^{\prime}\right)$ is maximally consistent, hence $(n, M)$ is consistent. Now tractability of the issue of consistency of a Horn representable generalized interval network follows easily:

Theorem 5 The issue of consistency of a Horn representable generalized interval network can be solved in polynomial time by means of the weak path-consistency algorithm.

\section{CONCLUSION}

We would like to emphasize that so far our main concern has been the connection between the syntactic concept of Horn representability and the geometric concepts of convexity, weak preconvexity and strong preconvexity. More precisely, we have given the set of all Horn representable relations between generalized intervals a simple characterization based on the concept of strong preconvexity. An advantage of this characterization is that it has allowed to present a simple proof that the issue of consistency of a Horn representable generalized interval network can be solved in polynomial time by means of the weak path-consistency algorithm.

Much remains to be done. We wish to investigate the question whether the class of binary relations between two generalized intervals generated by the set of all Horn representable relations between generalized intervals is the unique maximal tractable subclass among those which contain base relations. Future work also includes permitting the processing of metric constraints between generalized intervals, an important matter in the development of reasoning systems that are concerned with temporal information. 


\section{References}

[1] J. Allen, Maintaining knowledge about temporal intervals, Communications of the ACM, Vol. 26, pp. 832-843, 1983.

[2] P. Balbiani, J.-F. Condotta, L. Fariñas del Cerro, A model for reasoning about bidimensional temporal relations, KR'98, 1998, 124-130.

[3] P. Balbiani, J.-F. Condotta, L. Fariñas del Cerro, A new tractable subclass of the rectangle algebra, IJCAI-99, 1999, 442-447.

[4] P. van Beek, Reasoning about qualitative temporal information, Artificial Intelligence, Vol. 58, pp. 297-321, 1992.

[5] J.-F. Condotta, Problèmes de satisfaction de contraintes spatiales: algorithmes et complexité, Technical report, Institut de recherche en informatique de Toulouse, Toulouse, 2000.

[6] P. Ladkin, R. Maddux, The algebra of constraint satisfaction problems and temporal reasoning, Technical report, Kestrel institute, Palo Alto, 1988.

[7] G. Ligozat, On generalized interval calculi, AAAI-91, pp. 234-240, 1991.

[8] G. Ligozat, A new proof of tractability for ORD-Horn relations, AAAI-96, pp. 395-401, 1996.

[9] G. Ligozat, Generalized intervals: a guided tour, Technical report, Laboratoire d'informatique pour la mécanique et les sciences de l'ingénieur, Orsay, 1998.

[10] B. Nebel, H.-J. Bürckert, Reasoning about temporal relations: a maximal tractable subset of Allen's interval algebra, Journal of the ACM, Vol. 42, pp. 4366, 1995.

[11] J. Renz, Maximal tractable fragments of the region connection calculus: a complete analysis, IJCAI-99, pp. 448-454, 1999.

[12] J. Renz, B. Nebel, On the complexity of qualitative spatial reasoning: a maximal tractable fragment of the region connection calculus, Artificial Intelligence, Vol. 108, pp. 69-123, 1999.

[13] M. Vilain, H. Kautz, Constraint propagation algorithms for temporal reasoning, AAAI-86, pp. 377-382, 1986. 